\title{
La bulimia nerviosa y sus subtipos
}

\begin{abstract}
Myriam Sierra Puentes*
ABA Colombia, Asociación para el Avance de las Ciencias del Comportamiento Instituto San Bernardo de La Salle
\end{abstract}

Recibido: diciembre 17 de 2003

Revisado: enero 17 de 2005

Aceptado: enero 24 de 2005

\section{Resumen}

En este artículo se realiza una reseña sobre los trastornos de la alimentación, anorexia y bulimia, la relación que hacen los individuos con este comportamiento y la prevalencia, características y tratamientos más efectivos empleados en la bulimia nerviosa. Además, algunas aproximaciones para establecer subtipos de bulimia diferentes a los del DSM-IV e investigaciones sobre este trastorno del comportamiento alimentario, como la bulimia nerviosa.

Palabras clave: trastornos alimenticios, bulimia, procesos, subtipos.

\section{Abstract}

In this article, a brief description is realized about the disorders of feeding, anorexia and bulimia, the relationship made by individuals with this behavior, the prevalence, characteristics and the most effective treatments used for the bulimia nervous. And also some approximations to establish kinds of bulimia different of the DSM-IV and researches about this disorder of the feeding behavior which is the nervous bulimia.

Index terms: feeding disorders, bulimia, processes, kinds.

Correspondencia: Myriam Sierra Puentes. Correo electrónico: abacolombia@supercabletv.net.co 
A lo largo de la historia se han encontrado múltiples reportes de las molestias o exigencias que la alimentación puede acarrear en los individuos, bien sea porque se come poco, mucho o mal. Desde tiempos inmemorables, el comer ha sido un acto social que permite fortalecer relaciones, cerrar negocios, compartir con seres queridos e incluso mantener estatus, dependiendo de la cantidad de comida existente en los banquetes y la complexión física de los anfitriones o invitados; por ejemplo, la obesidad en otras épocas ha significado salud, riqueza, belleza, mientras que la delgadez se consideraba como fealdad, penuria o enfermedad; en la actualidad se ha invertido su significado social, se ve el rechazo a las personas con sobrepeso y se potencia un canon de belleza en competencia con nuestras características étnicas (Stice, Schupak-Neuberg, Shaw y Stein, 1994; Vitousek, Manke, 1994; Rozin y Fallon, 1998).

Los trastornos de alimentación constituyen uno de los problemas de salud de más rápido crecimiento en la población joven. Los niveles de prevalencia entre mujeres adolescentes oscila entre $1 \%$ y $3 \%$ (Attie y Brookis-Gunn, 1989; Fairburn, Hay y Welch 1993). Estos trastornos se caracterizan por alteraciones severas de la conducta alimentaria, y los que se presentan con más frecuencia son la anorexia nerviosa y la bulimia nerviosa. La anorexia se distingue por el rechazo a mantener el peso corporal en los valores mínimos normales, y la bulimia nerviosa por episodios recurrentes de voracidad o atracones, seguidos por conductas compensatorias inapropiadas como el vómito provocado, el abuso de fármacos laxantes, diuréticos $\mathrm{u}$ otros medicamentos, el ayuno o el ejercicio excesivo (Stice, Telch y Rizvi, 2000).

Una peculiaridad esencial de estos dos trastornos es la excesiva preocupación por la apariencia física, especialmente en lo referente a peso y talla y una alteración de la percepción de la forma y el peso corporales (Stice, Schupak-Neuberg, Shaw y Stein, 1994; Stice, Killen, Hayward y Taylor, 1998; Rozin y Fallon, 1998; Shisslak, Pazda y Crago, 1990).
A raíz del incremento en la incidencia, los trastornos de alimentación han sido objeto de amplia investigación en países desarrollados, especialmente los factores etiológicos, tanto desde perspectivas neurobiológicas como psicosociales; también se han diseñado diversos procedimientos de intervención para erradicar dicho trastorno (Garner y Garfinkel, 1997).

\section{Bulimia nerviosa}

Este trastorno constituye un preocupante problema de salud que afecta especialmente a la población femenina joven (Attie y Brookis-Gunn, 1989; Fairburn, Hay y Welch 1993).

\section{Descripción de bulimia nerviosa}

De acuerdo con el DSM-IV (APA, 1995), las características esenciales de la bulimia nerviosa consisten en atracones y en métodos compensatorios inapropiados (vómito provocado, el abuso de fármacos laxantes, diuréticos u otros medicamentos, el ayuno o el ejercicio excesivo) para evitar la ganancia de peso (utilizados en promedio dos veces a la semana durante un período de tres meses); además, la autoevaluación de los individuos con bulimia nerviosa se encuentra excesivamente influida por la silueta y el peso corporales. Basados en dicha preocupación, se tiende a presentar un patrón de ingesta hipocalórico o de dieta restrictiva. De acuerdo con el comportamiento, el DSM-IV describe dos tipos de bulimia. El purgativo incluye la autoprovocación del vómito o el uso de laxantes, diuréticos o enemas. El no purgativo utiliza otros tipos de conducta compensatoria como el ayuno o el exceso de ejercicio.

En cuanto al curso de desarrollo, la bulimia nerviosa se inicia generalmente al final de la adolescencia o al principio de la vida adulta. Los atracones suelen empezar después o durante un período de régimen dietético. El curso puede ser crónico o intermitente, con períodos de remisión que se alternan con atracones (APA, 1995). 


\section{Covariaciones}

La bulimia nerviosa covaría con trastornos del estado de ánimo, especialmente el trastorno distímico y el trastorno depresivo mayor, también con síntomas de ansiedad y con el abuso de sustancias psicoactivas y alcohol.

\section{Etiología}

Varias investigaciones sobre factores etiológicos han permitido identificar variables asociadas con el trastorno de bulimia nerviosa, tanto a un nivel neurobiológico como psicosocial, como dieta restrictiva, factores familiares (Graber y cols. 1994; Pike y Rodin, 1998; Wade y cols. 2000; Humphrey, 1989), alteraciones emocionales, déficit en autoestima, hábitos alimenticios, entre otros; actualmente no se ha establecido claramente la direccionalidad en dichas relaciones ni tampoco se han determinado los procesos psicológicos básicos que determinan los factores causales.

Una de las formulaciones más ampliamente aceptadas sobre el origen de la bulimia, planteada por el Grupo de Investigación de Oxford (Marcus y Fairburn, 1993), establece una secuencia en la cual un bajo nivel de autoestima lleva a una preocupación excesiva por el peso, generando dietas restrictivas, desequilibrio metabólico y episodios de atracones; éstos provocan conductas compensatorias, como vómito autoinducido, el cual finalmente cierra el círculo contribuyendo al déficit en autoestima. Sin embargo, no están claramente determinados ni se han investigado empíricamente los procesos psicológicos que llevan inicialmente al déficit en autoestima.

Por otra parte, hay datos investigativos y clínicos que señalan que, al igual que ocurre en otros trastornos de comportamiento, la bulimia es el resultado de la interacción de múltiples causas y de la participación de diversos procesos que, a pesar de resultar en las mismas manifestaciones, obedecen a causas diferentes, es decir, la bulimia no es un fenómeno unitario; personas que sufren del mismo trastorno pueden constituir subgrupos funcionalmente diferentes que responden de forma distinta a la intervención (Stice y Agras, 1999).

No obstante, los estudios que pretenden identificar diversos subtipos se han basado en variables clínicas o factorialmente derivadas, pero no han abordado los procesos psicológicos que a su vez podrían determinar dichas variables, de las cuales pueden derivar sub-tipos de bulimia, para dar paso a establecer modelos de tratamiento a través de los cuales se incremente la efectividad de los mismos y la disminución de la problemática que aqueja en aumento a la población actual. El DSM-IV plantea dos subtipos: el restrictivo y el purgativo para la bulimia nerviosa; pero estos subtipos no obedecen a una clasificación basada en factores psicológicos que puedan facilitar una clasificación más amplia que aborde un proceso de intervención enfocado a las variables que interactúan dentro de este trastorno.

\section{Intervención}

A lo largo de las últimas décadas, las posibles estrategias terapéuticas en bulimia han evolucionado encontrándose en el mercado un gran número de ellas, según Fernández (1997), citado por Turòn (1997), las formas de terapia más aplicadas son cinco: 1) psicoterapia individual, 2) grupo de autoayuda, 3) hospitalización, 4) tratamiento farmacológico y 5) tratamiento grupal.

Desde una perspectiva de intervención, se han aplicado diversos formatos de tratamiento para tratar de restablecer los patrones normales de alimentación, incluyendo medicación (Garnre y Garfinkel, 1997), terapia familiar, terapia psicodinámica y terapia cognoscitiva conductual -TCC- (Wilson, 1996; Wilson y Fairburn, 1993). De todos estos métodos, se ha concluido que esta última es la que mejores resultados ofrece a la población aquejada por la bulimia nerviosa.

En más de 30 estudios se ha encontrado que la TCC (Wilson y Fairburn, 1993) es el método más 
efectivo y el que mejores resultados ha producido en este trastorno en comparación con otros métodos como terapia no directiva, terapia psicodinámica, manejo de estrés, medicación antidepresiva y ausencia de terapia (Agras y cols., 1997; Wilson y cols., 1993). De hecho, la TCC de la bulimia nerviosa se ha reconocido como uno de los tratamientos empíricamente validados en el campo de la psicología de la salud.

Al revisar los datos de los estudios más rigurosos, se encontró un porcentaje promedio de reducción de atracones entre el $93 \%$ y el $73 \%$ y de conductas compensatorias (por ejemplo, vómito autoinducido) entre el $94 \%$ y $77 \%$ (Wilson y cols., 1993).

Pero a pesar de estos resultados replicados en distintos centros de investigación, en la población colombiana no se han realizado investigaciones de procesos ni de resultados que permitan identificar los factores causales que más afectan a las personas que presentaron bulimia ni tampoco estudios controlados que permitan validar y estandarizar procedimientos de evaluación y de intervención que sean aplicables a las condiciones propias del ámbito colombiano.

\section{Una aproximación a subtipos de bulimia nerviosa}

Los subtipos planteados por el DSM IV son el purgativo y el no purgativo, descritos anteriormente; adicional a éstos se han encontrado otros subtipos a través de investigaciones, tratando de establecer factores psicológicos que identifiquen la adquisición y mantenimiento que operen en la bulimia nerviosa de una forma predominante y constante en todos los individuos que la presentan; entre las investigaciones se encuentra una que plantea dos dimensiones de la restricción dietética y afecto negativo de Stice y Agras (1999), quienes proponen que los modelos etiológicos de la bulimia se centran en la dieta y el afecto negativo.

La teoría del afecto negativo postula que una perturbación emocional elevada incrementa la pro- babilidad que una persona inicie la alimentación compulsiva (León y cols., 1995). Teóricamente, los individuos con un afecto negativo elevado comen compulsivamente debido a que creen que esto les provee comodidad y los distrae de sus emociones negativas.

Aún la investigación no ha subdividido a los individuos con bulimia, ni han tenido en cuenta si encajan en el perfil dietético versus afecto negativo. Este estudio subdividió 265 mujeres bulímicas entre las dimensiones dietética y depresiva, probando que los subtipos mostraban diferencias en la patología de la alimentación, los correlatos clínicos y la respuesta al tratamiento. Un análisis de clusters reveló un subtipo dietético (dietario) puro (62\%) y un subtipo mezclado dietético-depresivo (38\%). Mientras las mujeres con bulimia dietéticas y las dietéticas-depresivas mostraron niveles similares de comportamientos bulímicos; el segundo grupo -afecto negativo- reportó más obsesiones con la alimentación y con el peso, desajuste social, altas tasas de estado de ánimo depresivo, ansiedad, alimentación, control de impulsos, desórdenes de personalidad y una respuesta pobre al tratamiento. Los resultados sugieren que la dieta es una característica central de la bulimia, pero el afecto depresivo ocurre sólo en un subconjunto de casos. Sin embargo, la combinación de dieta y afecto depresivo parece ser señal de una variante más severa de la bulimia (Stice y Agras, 1999).

Los mismos autores definen un subtipo dietético (dietario) y un subtipo mezclado dietético-depresivo; la definición de estos subtipos de bulimia se basa en los siguientes modelos: por una parte, el modelo de restricción que plantea que la dieta elevada resulta en gran riesgo para la alimentación compulsiva (Heatherton y Polivy, 1992, citados por Stice y Agras 1999). Teóricamente, la dieta incrementa las posibilidades de que un individuo presente alimentación compulsiva como un esfuerzo para contrarrestar los efectos de la deprivación calórica. Es más, las transgresiones de las reglas de una dieta estricta pueden resultar en una alimentación desinhibida debido a los efectos de la violación de la abstinencia. 
El modelo de doble vía de la bulimia nerviosa propone que tanto la restricción dietética como el afecto negativo pueden jugar un papel en la promoción de la patología bulímica, en la que el individuo puede iniciar la alimentación compulsiva debido tanto a la dieta o a las distorsiones afectivas o a alguna combinación de estos factores (Stice, 1994, citada por Stice, 1999). Por lo tanto, el modelo de doble vía propone que estos dos factores sirven como los mecanismos finales por los cuales las presiones socioculturales más generales, como la presión percibida para ser delgado y la insatisfacción corporal, promueven el desarrollo de las conductas bulímicas.

La dieta se correlacionó con los síntomas bulímicos futuros. León y cols. (1995) predijeron el ataque de la patología bulímica entre los individuos asintomáticos. De igual manera, el afecto negativo se correlacionó con los síntomas bulímicos futuros; León y cols. (1993) predijeron el ataque de la alimentación compulsiva y la purga entre los individuos libres de desorden de la alimentación.

Es importante resaltar que este estudio es el primero que ha intentado subdividir a los individuos bulímicos a lo largo de las dimensiones de dieta y depresión. Los autores resaltan que los hallazgos obtenidos no deben interpretarse como indicadores, sino que la dieta y el afecto depresivo son las únicas o las mejores dimensiones para subdividir a los individuos con bulimia. Se resalta que pueden haber otras variables que no fueron examinadas aquí, como el tiempo de presentación del trastorno, y que también pueden ser útiles para subdividir a los individuos bulímicos. Se sugiere que los estudios longitudinales con el tiempo son necesarios para el propósito de predecir el inicio o mantenimiento del comportamiento bulímico. Los resultados sugieren que esto podría ser útil para considerar la restricción dietética y el afecto negativo en las investigaciones que examinen la etiología de la bulimia.

La investigación ha señalado que una variedad de afectos negativos precipita la alimentación compulsiva, incluyendo el estrés, la ansiedad y el estado de ánimo negativo general (Davis, Freeman y Garner, 1991; Elmore y De Castro, 1990; Lingswiler, Crowther, y Stephens, 1989), citados por Stice (1999).

Una implicación importante, resultado de este estudio, es que la TCC típico para la bulimia nerviosa puede reducir la frecuencia de la alimentación compulsiva y la purga, pero puede ser menos probable para reparar las distorsiones afectivas y el deterioro social relacionado. Por lo anterior, la TCC puede ser menos efectiva para individuos con el trastorno bulímico dietético depresivo, debido a que ellos tienen atracones para regular sus problemas emocionales y estas distorsiones afectivas no son intervenidas directamente en este tratamiento.

Serán necesarios estudios longitudinales para explorar la significación del pronóstico de estos dos tipos de bulimia para los cambios en las actitudes y comportamientos bulímicos, los correlatos clínicos, el funcionamiento social y el diagnóstico comórbido a lo largo del tiempo.

Los datos obtenidos sugieren que pueden ser dos subtipos distintos de bulimia con diferentes características asociadas y diferentes proporciones de patología comórbida. La investigación futura también debe probar si los factores de riesgo son similares para estos dos aparentes subtipos de bulimia. Teniendo en cuenta las implicaciones del tratamiento, el hallazgo de una proporción considerable de los individuos con bulimia parece mostrar un afecto depresivo marcado, esto puede explicar por qué los individuos no responden a la TCC, debido a que este tratamiento no se dirige explícitamente a los problemas del estado de ánimo. Estos hallazgos pueden sugerir que es importante dirigir los problemas afectivos en un subconjunto de individuos bulímicos tratándolos directamente con terapia cognoscitiva o antidepresivos, o enseñándoles habilidades de regulación del afecto más adaptativas que puedan reemplazar funcionalmente los atracones como una respuesta de afrontamiento. Sin embargo, debido a que los individuos depresivos también muestran una dieta elevada, puede ser aconsejable seguir un tratamiento enfocado en el afec- 
to con una intervención cognoscitivo conductual estándar para la bulimia. Finalmente, la proporción de éxito del tratamiento para la bulimia nerviosa puede aumentarse si se atienden las variaciones en la presentación clínica del desorden.

A la par de esta investigación, se han realizado otras aproximaciones a la clasificación de la bulimia en subtipos, ya que a la luz de la literatura se evidencia la importancia de hacer una clasificación más detallada, con el fin de realizar un proceso de evaluación, identificación e intervención precisa que eventualmente pueda incrementar la efectividad de los tratamientos, aumentando la probabilidad de éxito en las intervenciones.

\section{Otros niveles de investigación}

Dada la seria amenaza para la salud, la bulimia nerviosa, al igual que otros desórdenes de alimentación, ha sido objeto de investigación exhaustiva en múltiples centros de investigación en el contexto internacional y se han identificado diferentes factores causales a nivel neurobiológico, psicológico y social. A nivel neurobiológico, se han identificado factores nutricionales, eventos fisiológicos periféricos y procesos centrales neuroquímicos, que indican la posibilidad de que al menos en algunos casos los desórdenes alimenticios pueden surgir como resultado de procesos neurobiológicos endógenos producidos por un déficit en la regulación del sistema de control de apetito; igualmente, otras investigaciones han identificado factores de riesgo o predisponentes en el ámbito familiar, como tener antecedentes de familiares con problemas de alimentación o excesiva preocupación por el peso y patrones perfeccionistas y de alta exigencia en los padres (Graber y cols., 1994; Pike y Rodin, 1998; Wade y cols., 2000; Humphrey, 1989).

Por otro lado, gran parte de la investigación se ha centrado en identificar características propias de las personas con bulimia, así como su relación con otras entidades diagnósticas. En primer lugar, en cuanto a variables demográficas y de género, se ha encontrado mayor incidencia en mujeres; en relación con la edad, se ha presentado entre 13 y 24 años.

En cuanto a características personales, los investigadores han encontrado una alta variedad de factores a partir de variables de personalidad, déficit en habilidades sociales y dificultades en las relaciones interpersonales, patrones de pensamiento perfeccionistas y dicótomos. Aunque no hay muchos estudios controlados sobre factores causales en nuestro medio, Rodríguez y Gempeler (1999) en un estudio con 32 pacientes de sexo femenino, y mediante la aplicación de un conjunto de medidas sobre imagen corporal, creencias sobre el comer y autoimagen, entre otras, identificaron nueve posibles factores externos precipitantes asociados con el trastorno, éstos son: burlas (críticas o adulación excesivas relacionadas con el cuerpo), sobrepeso real durante la prueba, sobrepeso percibido, viajes sin los padres (campamentos de verano), formas de maltrato diferentes al físico, separación de los padres (después de estar casados durante más de cinco años), abuso sexual franco, matrimonio de uno de los padres (después de separase del padre biológico), proceso de crecimiento y maduración rápi$\mathrm{da}$, concluyendo que lo que denominan "corporalidad" constituía el eje fundamental del trastorno.

A pesar de que en los múltiples estudios referenciados se ha identificado un déficit en autoestima o autoimagen como factor asociado y supuestamente causal (Vohs y cols., 1999), no se ha establecido una clara relación de direccionalidad; tampoco se han descrito los factores que determinan el déficit de autoestima; de hecho, en muchos estudios se toma como un constructo causal (Ruderman y Besbeas, 1992). El estado afectivo negativo asociado con el bajo nivel de autoestima ha llevado a los investigadores a proponer un modelo de intervención con dieta restrictiva (Stice y Agras, 1999); a su vez, la dieta restrictiva se ha relacionado con los atracones (Lowe y cols., 1998). 
Los anteriores hallazgos han llevado a los principales investigadores en bulimia a proponer un modelo en espiral de la bulimia nerviosa que tiene varias etapas (Fairburn, Marcus y Wilson, 1993). Inicialmente, la persona tiene una mala autoimagen y un alto grado de insatisfacción con su aspecto físico en referencia con peso y talla.

A pesar de la controversia sobre la naturaleza misma de la bulimia en relación con otros problemas de alimentación, como la anorexia nerviosa o el desorden de comer en exceso, se han realizado estudios multifactoriales que han apoyado la existencia de la bulimia como un trastorno diferente e independiente de otros problemas de alimentación; además, se encuentran datos clínicos e investigativos que indican que la bulimia es el resultado de la interacción de causas diversas, como la investigación mencionada anteriormente, que llevan a la participación de distintos procesos psicológicos que pueden producir resultados aparentemente similares, pero son procesos particulares. En otras palabras, de acuerdo con esta posición, a pesar de que la bulimia constituye una categoría diferente de otras disfunciones de la conducta de alimentación, en sí misma no es una clasificación unitaria, sino que está formada por diversos subtipos funcionalmente diferentes que mostrarían un resultado particular en la intervención (Stice y Agras, 1999); no obstante, al igual que ha ocurrido con el concepto de autoestima, estos factores son a su vez el resultado de la participación de procesos psicológicos básicos que no se han identificado adecuadamente.

Para realizar una intervención eficaz e identificar las posibles clasificaciones o subtipos de bulimia nerviosa, autoestima y otro trastornos, se necesita un esquema de formulación que permita identificar y explicar adecuadamente los procesos psicológicos que dan lugar a las manifestaciones clínicas, con el fin de no centrarse únicamente en describir la regularidad entre los sucesos conductuales y ambientales de los trastornos, en especial de la bulimia nerviosa. Este esquema permitirá la predicción de aparición de las conductas o las características topográficas de las respuestas que faciliten posteriormente realizar un análisis de contingencia y que también atienda los diferentes niveles de respuesta que dependen de distintos sistemas de concordancia, estableciendo de manera clara y consistente las relaciones que tienden a covariar y a interactuar entre sí en esta problemática, logrando así determinar en el individuo cuál es el componente de su comportamiento que debe modificar e indicarle cuál es el mejor método para hacerlo.

En cuanto al proceso de formulación, las investigaciones han llevado a Castro y Ángel (1998) a plantear un modelo jerárquico de organización conductual que diferencia los niveles de establecimiento de relaciones entre distintos acontecimientos; estos niveles corresponden a procesos dinámicos donde se relacionan eventos ambientales y conductuales que conforman complejas redes causales que permiten ejecutar un trabajo en el que efectivamente se identifiquen e intervengan directamente estos procesos, y su éxito, claro está, depende de la modificación de los mecanismos que producen la conducta en particular.

En otras palabras, el enfoque por procesos psicológicos permite identificar las relaciones o las leyes que tienden a covariar y a interactuar entre sí en una problemática particular, permitiendo establecer aquellos procesos que no son reductibles en otros (procesos básicos), identificando las leyes que los conforman y describiendo la clase de interacción que permite establecer las redes complejas que los llevan a procesos de segundo orden, facilitando de esta manera la explicación e intervención de la conducta problema.

De acuerdo con este modelo, los comportamientos relacionados con la bulimia nerviosa están estructurados teniendo en cuenta la acción de diversos procesos interdependientes que están organizados jerárquicamente en distintos niveles: procesos básicos (biológico, aprendizajecognoscitivo y motivacional), procesos derivados (emocional y afectivo), covariaciones y problemas específicos. 
En el primer nivel, se plantean tres procesos bási$\cos$ no reducibles a otro nivel superior, que conforman la red de causación múltiple del comportamiento.

El proceso biológico se refiere a una serie de variables de estado, se relaciona con la materia de que está hecho el organismo, incluyendo factores genéticos, morfológicos y de funcionamiento de los sistemas de órganos a través de mecanismos bioquímicos, neuroendocrinos, eléctricos y metabólicos, entre otros. De acuerdo con la literatura sobre bulimia nerviosa, se encuentran bajos niveles de serotonina, pero que es preciso determinar si corresponde a una causa o una consecuencia del desorden.

El proceso aprendizaje-cognoscitivo se refiere al conjunto de relaciones y principios que determinan la adquisición de nuevos repertorios y el cambio de comportamientos de los organismos a través del establecimiento de relaciones entre patrones de acontecimientos ambientales y patrones de acontecimientos conductuales, por medio de mecanismos como condicionamiento clásico, condicionamiento operante (selección por contingencias), aprendizaje observacional, control instruccional (reglas), entre otros.

El proceso motivacional hace referencia a la relación que existe entre el comportamiento y sus consecuencias que, junto con las alternativas conductuales disponibles, le permite al individuo, por una parte, predecir y, por la otra, ejercer control sobre su propio comportamiento y sobre su ambiente.

\section{Conclusiones}

Se evidencia que la bulimia nerviosa ha sido investigada desde diferentes puntos de vista a raíz de su aumento en la población, tratando de identificar los factores causales y los factores de mantenimiento, pero pocas de estas investigaciones apuntan a subdividir este trastorno. Adicionalmente, no se evidencia una teoría que apunte a clasificar a los individuos que presentan bulimia nerviosa a través de los procesos psicológicos que interactúan en la adquisición y mantenimiento; si se identificaran claramente estos procesos con una evaluación sistemática, se facilitaría un proceso de intervención más efectivo y posiblemente se incrementarían los niveles de efectividad de los tratamientos, como la TCC.

\section{Referencias}

Agras, S. W., Telch, C. F., Arnow, B., Eldredge, K. y Marnell, M. (1997). One-Year Follow-Up of Cognitive-Behavioral Therapy for Obese Individuals With Binge Eating Disorder. Journal of Consulting and Clinical Psychology, 65, 343-347.

Attie, I., Brooks-Gunn, J. (1989). Development of Eating Problems in Adolescent Girls A Longitudinal Study. Developmental Psychology, 25, 70-79.

DSM - IV Manual Diagnóstico y Estadístico de los Trastornos Mentales, (1995). American Psychiatric Association (4a ed.). Barcelona: Masson.

Garner, D. M. (1991). Eating Disorders Inventory - 2. Odessa, FL: Psychological Assessment Resources.

Garner, D. M.\& Garfinkel, P. E. (1997). Handbook of Treatment for Eating Disorders. $2^{\text {nd }} \mathrm{Ed}$. The Guilford Press: New York.

Graber, J. A., Brooks-Gunn, J., Paikoff, R. L. y Warren, M. P. (1994). Prediction of Eating Problems: An 8-Year Study of Adolescent Girls, Developmental Psychology. 30, 823834.

Grissett, N. I. y Norvell, N. K. (1992). Perceived Social Support, Social Skills, and Quality of Relationships in Bulimic Women. Journal of Consulting and Clinical Psychology, 60, 293299. 
Humphrey, L. L. (1989). Observed Family Interactions Among Subtypes of Eating Disorders Using Structural Analysis of Social Behavior. Journal of Consulting and Clinical Psychology, 57, 2, 206-214.

León, G. R., DE A. Fulkerson, J., Perry, C. L. y Temprano - Zald M. B. (1995). Journal of Abnormal Psychology, 104, 1, 140-149.

Pike, K. M. y Rodin, J. (1998). Madders, daughters and eating disorders. Journal of abnormal psychology, 100,198-204.

Rozin, P. y Fallon, A. (1998). Body Image, Attitudes to Weight, and Misperceptions of Figure Preferences of the Opposite Sex A Comparison of Men and Women in Two Generations. Journal of Abnormal Psychology, 97, 342-345.

Shisslak, C. M., Pazda, S. L. y Crago, M. (1990). Body Weight and Bulimia as Discriminators of Psychological Characteristics Among Anorexic, Bulimic, and Obese Women. Journal of Abnormal Psychology, 99, 380384.

Stice, E. y Agras, W. S. (1999). Subtyping Bulimic Women Along Dietary Restraint and Negative Affect Dimensions. Journal of Consulting and Clinical Psychology, 67, 4, 460-469.

Stice, E., Killen, J. D., Hayward, C. y Taylor, C. B. (1998). Support for the Continuity
Hypothesis of Bulimic Pathology. Journal of Consulting and Clinical Psychology, 66, 784790.

Stice, E., Schupak-Neuberg, E., Shaw, H. E. y Stein, R. I. (1994). Relation of Media Exposure to Eating Disorder Symptomatology An Examination of Mediating Mechanisms. Journal of Abnormal Psychology, 103, 4, 836-840.

Stice, E., Telch, C. F., y Rizvi, S. L. (2000). Development and Validation of the Eating Disorder Diagnostic Scale A Brief Self-Report Measure of Anorexia, Bulimia, and BingeEating Disorder. Psychological Assessment, 12,2,123-131.

Turón, Gil, J. V. (1997). Los Trastornos Alimenticios. Ed.Masson.

Wade, T. D., Bulik, C. M., Sullivan, P. F., Neale, M. C. y Kendler K. S. (2000). The Relation Between Risk Factors for Binge Eating and Bulimia Nervosa A Population-Based Female Twin Study. Health Psychology, 19, 115-123.

Wilson G. T. y Fairburn, C. G. (1993). Cognitive Treatments for Eating Disorders. Journal of Consulting and Clinical Psychology, 61, 261269.

Wilson, T. (1996). Treatment of Bulimia Nervosa: When CBT fails. Behavior Research and Therapy, 34, 3. 197 - 212. 